\title{
Prevalence of co-existing autoimmune disease in juvenile idiopathic arthritis: a cross-sectional study
}

Teresa A. Simon ${ }^{1 *}$, Gowri Priya Harikrishnan², Hugh Kawabata', Sanket Singhal ${ }^{2}$, Hermine I. Brunner ${ }^{3}$ and Daniel J. Lovell ${ }^{3}$

\begin{abstract}
Background: Many autoimmune diseases share common pathogenic mechanisms, cytokine pathways and systemic inflammatory cascades; however, large studies quantifying the co-existence of autoimmune diseases in patients with juvenile idiopathic arthritis (JIA) have not been conducted.
\end{abstract}

Methods: We performed a cross-sectional study using two United States administrative healthcare claims databases (Truven Health MarketScan ${ }^{\oplus}$ Commercial Database and IMS PharMetrics database) to screen for the prevalence of multiple autoimmune diseases in patients with JIA and in a control group with attention deficit hyperactivity disorder (ADHD). Patients with a diagnosis code for JIA or ADHD between January 1, 2006 and September 30, 2017 were separated into two age cohorts ( $<18$ and $\geq 18$ years) and matched (maximum 1:5) based on age, sex, number of medical encounters, and calendar year of diagnosis. The prevalence rates of 30 pre-specified autoimmune diseases during the 12-month periods before and after diagnosis were compared.

Results: Overall, 29,215 patients with JIA and 134,625 matched control patients with ADHD were evaluated. Among patients in the MarketScan database, 28/30 autoimmune diseases were more prevalent in patients with JIA aged < 18 years and 29/30 were more prevalent in patients aged $\geq 18$ years when compared with a matched cohort of patients with ADHD. In the PharMetrics database, 29/30 and 30/30 autoimmune diseases were more prevalent in patients with JIA aged $<18$ and $\geq 18$ years, respectively, compared with a matched cohort of patients with ADHD. Among patients with JIA aged < 18 years, the greatest odds ratios (ORs) were seen for Sjögren's syndrome/sicca syndrome and uveitis. Among patients aged $\geq 18$ years in the MarketScan database, the greatest ORs were recorded for uveitis. Data from the PharMetrics database indicated that the greatest ORs were for uveitis and chronic glomerulonephritis.

Conclusions: Patients with JIA are more likely to have concurrent autoimmune diseases than matched patients with ADHD. Having an awareness of the co-existence of autoimmune diseases among patients with JIA may play an important role in patient management, treatment decisions, and outcomes.

Trial registration: Not applicable.

Keywords: ADHD, Comorbidity, Co-existing autoimmune disease, Epidemiology, Juvenile idiopathic arthritis, Prevalence

\footnotetext{
* Correspondence: teresa.simon1@verizon.net

'Bristol Myers Squibb, Princeton, NJ, USA

Full list of author information is available at the end of the article
}

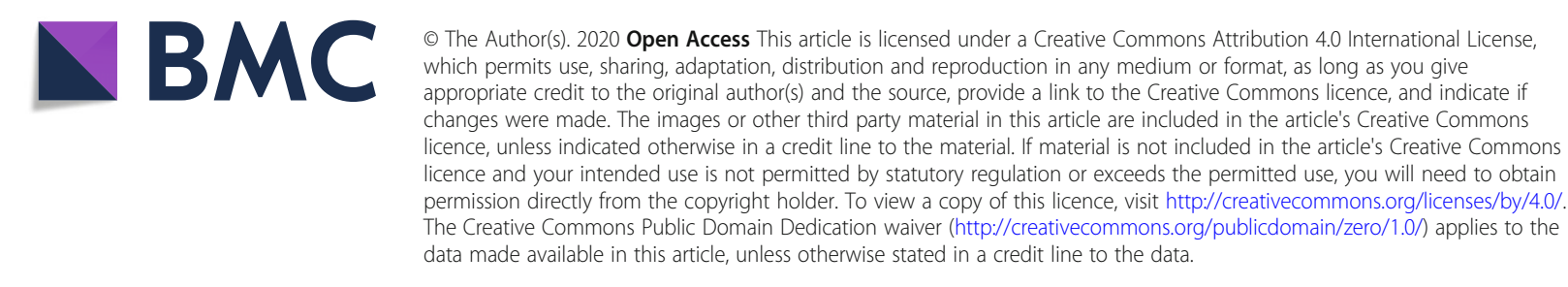




\section{Background}

Autoimmune diseases are the most prevalent disease conditions reported in the United States (US) [1]. The overall estimated prevalence rate of autoimmune disease is $3.1 \%$ in the US [2] and $7.6-9.4 \%$ in Europe [3], depending on the source and if correction for underascertainment is used. Research has demonstrated that the incidence and prevalence of autoimmune diseases have been steadily and significantly rising over the past three decades [4]. This is of particular concern as autoimmune diseases have been associated with increases in morbidity and mortality $[5,6]$.

There are over 100 known autoimmune diseases [7] and many exhibit shared pathogenic mechanisms [8], reflecting a complex interaction between environmental and genetic factors [9]. Genome-wide association studies have increased the understanding of the shared cytokine pathways and systemic inflammatory cascades characteristic of autoimmune diseases $[8,9]$. Despite being in an era of electronic medical records and integrated care, the US remains mostly a siloed healthcare system [10]. Therefore, as autoimmune diseases span a number of medical specialties, it is important to raise awareness of those that overlap disciplines in order to improve patient care.

Juvenile idiopathic arthritis (JIA) is the most common chronic pediatric rheumatic disease and is characterized by joint inflammation that often leads to joint damage, chronic pain and disability $[11,12]$. The term 'JIA' encompasses seven clinically heterogeneous groups of arthritides of unknown cause in children [11]. JIA is not confined to childhood, with more than a third of patients continuing to have episodes of active inflammation during their adult years [13, 14]. Several studies have reported the prevalence of JIA; however, there is limited knowledge about the frequency of the cooccurrence of other autoimmune diseases in patients with JIA $[15,16]$, with the exception of uveitis, which is known to occur in $12-31 \%$ of patients with JIA $[17,18]$. In addition, for JIA, the majority of publications are case reports [19-21] or small clinical/hospital studies [22-28] involving one or a select few autoimmune diseases. These studies show that co-existing autoimmune diseases, particularly thyroid conditions, are common in patients with JIA. Previous studies have evaluated the co-existence of other autoimmune diseases in patients with psoriasis [29] and adult patients with rheumatoid arthritis (RA) [15] and multiple sclerosis [30].

Comorbid conditions in patients with JIA, including coexisting autoimmune diseases, negatively impact quality of life, disability, and mortality [31, 32]. In a single-center study of 79 patients with JIA, $10.1 \%$ had one additional autoimmune disease and 5.1\% had two [28]. As such, understanding the presence of overlapping autoimmune diseases is important in developing treatment strategies, particularly as some autoimmune diseases can arise or be worsened by common treatments, such as tumor necrosis factor inhibitors, which are commonly used in the treatment of diseases such as JIA and RA [8,33, 34].

The aim of this large, cross-sectional study was to quantify the co-occurrence of autoimmune diseases in patients with JIA. In addition, this study evaluated the distribution of these autoimmune diseases based on age $(<18$ and $\geq 18$ years). In a database study, among patients with JIA who transferred from pediatric to adult rheumatology care after the age of 14 years, the median age at transfer was 18 years [35]. To address potential observation bias, the prevalence rates of co-existing autoimmune diseases were also evaluated in patients with attention deficit hyperactivity disorder (ADHD, a common disorder generally diagnosed in childhood that often persists into adulthood [36]), who have similarly frequent interactions with the healthcare system (e.g. healthcare encounter) as do patients with JIA [37].

\section{Methods \\ Study design}

This was a large, retrospective, cross-sectional study using two US administrative healthcare claims databases: the Truven Health MarketScan ${ }^{\circ}$ Commercial Database $\left(\right.$ MarketScan $^{\circ}$ ) and the IMS PharMetrics database (PharMetrics). MarketScan includes data from 2006 onward for over 70 million privately insured patients under the age of 65 years. PharMetrics contains claims information on approximately 55 million patients from over 90 managed care plans and other sources from 2007 onward. These databases contain insurance claim information that includes anonymized information on diagnoses associated with physician visits, medical procedures, hospitalizations, prescribed medications, and laboratory tests for insured individuals.

A common data model was used to standardize the data structure and terminologies of the two databases in order to minimize the need for customizations and allow the proposed analytic methods to be applied systematically. The use of this model allows the source data to be represented more reliably [38].

The study was conducted in accordance with the International Society for Pharmacoepidemiology Guidelines for Good Pharmacoepidemiology Practices [39], applicable regulatory requirements and the principles of the Declaration of Helsinki. No new studies with human or animal subjects are reported in this article. Review and approval by ethics committees and patient informed consent were not required for this study because data were anonymized at the source. 


\section{Patients}

Patients with a diagnosis code for JIA or ADHD between January 1, 2006 and September 30, 2017 were included in this study; this was not necessarily the first time the patient was diagnosed with JIA or ADHD. Patients were required to have two occurrences of an International Classification of Diseases, Ninth Revision, Clinical Modification (ICD-9-CM) diagnosis code of 714.3x or International Classification of Diseases, Tenth Revision, Clinical Modification (ICD-10-CM) diagnosis code of M08.x for JIA and 314.0x (ICD-9-CM) and F90.x (ICD-10-CM) for ADHD on separate occasions within a 90-day period. At least 1 year of continuous health plan enrollment before the qualifying JIA or ADHD diagnosis code and 1 year after the diagnosis code were required for inclusion in this study. Patients were stratified into four cohorts: < 18-year-old patients with JIA, $\geq 18$-year-old patients with JIA, <18year-old patients with $\mathrm{ADHD}$, and $\geq 18$-year-old patients with ADHD. Age was categorized based on age of the patient at the time the qualifying JIA/ADHD diagnosis code was reported. Patients with both JIA and ADHD or $>3$ medical encounters listed in 1 day were excluded.

\section{Outcomes}

Prevalence rates of 30 common autoimmune conditions (Supplementary Table 1) were investigated among patients with JIA or ADHD during the 1-year baseline (when the first qualifying JIA/ADHD diagnosis code was reported) and 1-year follow-up periods. RA was included as an outcome not to observe if it co-existed with JIA, but to ascertain whether a greater proportion of patients with JIA aged $\geq 18$ years, compared with those $<18$ years, also received a diagnosis of RA.

\section{Statistical analyses}

Patients with JIA were frequency matched to patients with ADHD for each combination of the following characteristics: age ( \pm 2 years), sex, number of medical encounters (in-patient, out-patient, and emergency room visits), and calendar year of diagnosis ( \pm 1 year). For each patient with JIA, up to 5 matched patients with ADHD were identified. Matching was carried out with replacement for the ADHD group; patients with ADHD could be matched to a maximum of 7 (PharMetrics) or 8 (MarketScan) patients with JIA.

Descriptive statistics were used to analyze baseline demographic information, comorbid conditions, and medication use. The co-occurrence of autoimmune diseases was studied using a univariate analysis to understand their individual association with JIA/ADHD. Data are presented as matched prevalence rates, 95\% confidence intervals and odds ratios (ORs) for patients who had two relevant ICD-9CM or ICD-10-CM diagnosis codes for any of the 30 prespecified autoimmune diseases within 1 year before and after the qualifying JIA/ADHD diagnosis code was recorded. Descriptive statistics (\%) were used to analyze the reported prevalences of RA, ankylosing spondylitis, psoriatic arthritis, dermatomyositis, systemic lupus erythematosus (SLE), and sarcoidosis as a diagnosis code for these diseases does not represent co-existence, but more likely misclassification. Results presented by age group are shown for the patients' ages at the time of the study.

\section{Results}

Patient disposition and baseline characteristics

A total of 30,779 patients with JIA and 2,515,136 patients with ADHD were eligible for inclusion across the two databases (Fig. 1). After matching and removal of those with excess matches $(>5)$ or excess encounters (> 3), 29,215 patients with JIA and 134,625 patients with ADHD were analyzed. Across both databases and age cohorts, $100 \%$ of patients with JIA were included.

The baseline characteristics of each individual cohort were similar before (Supplementary Table 2) and after matching (Table 1), with the exception of sex and number of medical encounters. These differences were accounted for with the frequency matching analyses. In the matched patients, across all cohorts, approximately three-quarters of patients were female (range: 69-78\%), with a mean age of $10.5-11$ years in the $<18$ years cohort and 34-37 years in the $\geq 18$ years cohort at the time of the qualifying JIA/ADHD diagnosis code (Table 1). Across both age cohorts, a greater proportion of patients with JIA were receiving disease-modifying antirheumatic drugs (DMARDs), corticosteroids, or non-steroidal antiinflammatory drugs compared with those with ADHD. Among patients with JIA, $23-25 \%$ of those aged $<18$ years and $52-54 \%$ aged $\geq 18$ years also had a diagnosis of RA. This is expected, as non-pediatric rheumatologist physicians may give a diagnosis of RA, in particular when a patient with JIA moves from pediatric care to an adult healthcare setting [41, 42]. Diagnoses of psoriatic arthritis and ankylosing spondylitis, respectively, were reported in 3 and $1 \%$ of patients with JIA aged $<18$ years and $1-2$ and $2 \%$ aged $\geq 18$ years. Psoriatic arthritis and ankylosing spondylitis were not considered as co-existing autoimmune diseases because the diagnosis codes for these autoimmune diseases may be used for specific subcategories of JIA. Dermatomyositis, SLE, and sarcoidosis, respectively, were reported in $0.5 \%, 0.8-0.9 \%$, and $0.1 \%$ of patients with JIA aged < 18 years and $0.3 \%, 2-3 \%$ and $0.4 \%$ aged $\geq 18$ years. As these diseases present with symptoms that overlap with JIA, which may lead potential errors in diagnoses codes, these diseases were not considered co-existing.

All patient characteristics were similar between both healthcare claims databases, except number of medical encounters, which were higher in the PharMetrics cohort. 


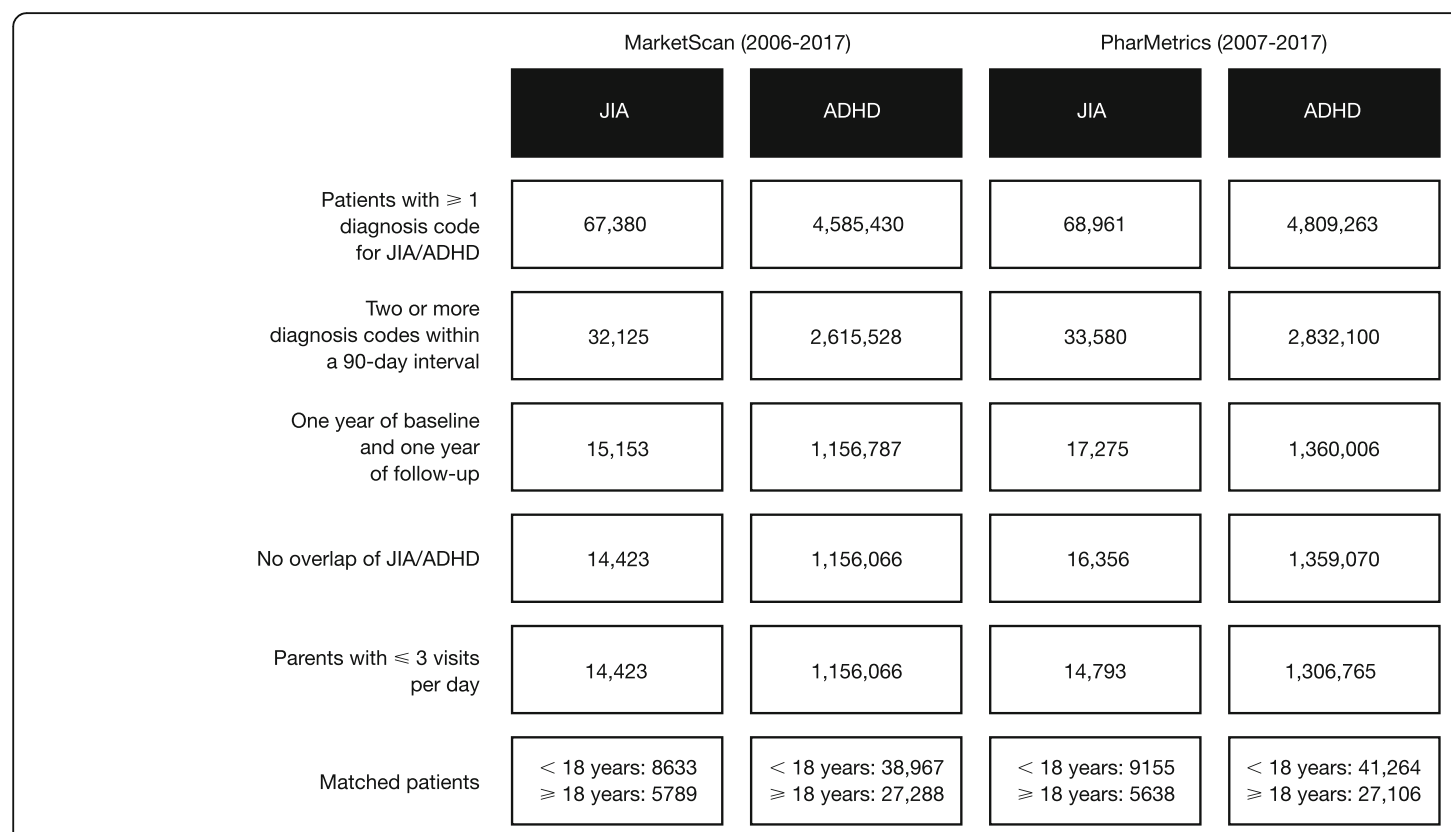

Fig. 1 Patient disposition. Cells show number of patients. ADHD attention deficit hyperactivity disorder, JIA juvenile idiopathic arthritis, MarketScan Truven Health MarketScan ${ }^{\oplus}$ Commercial Database, PharMetrics IMS PharMetrics database

Differences in baseline comorbid conditions were noted between patients of all ages with JIA and ADHD (Table 1): patients with JIA were more likely to have anemia, whereas patients with ADHD were more likely to have anxiety or depression. In both the JIA and ADHD cohorts, patients in the $<18$ years cohorts showed a lower rate of all comorbid conditions, except asthma, and also lower rates of DMARD and corticosteroid use compared with the $\geq 18$ years cohort. The total number of co-existing autoimmune diseases identified among the patients with JIA was greater compared with those with ADHD for patients of all ages. Both patients with JIA and patients with ADHD aged $\geq 18$ years were more likely to have more than one autoimmune disease than patients $<18$ years with the same conditions.

\section{Prevalence}

Overall, the prevalence rates of the pre-specified autoimmune diseases evaluated were higher among patients with JIA than among matched patients with ADHD (Figs. 2 and 3, Table 2), with few differences between the two databases. Among patients with JIA in MarketScan, $28 / 30$ autoimmune diseases were more prevalent in patients with JIA aged < 18 years and 29/30 were more prevalent in patients aged $\geq 18$ years, when compared with a matched cohort of patients with ADHD. In PharMetrics, 29/30 and 30/30 autoimmune diseases were more prevalent in patients with JIA aged $<18$ and $\geq 18$ years, respectively, compared with a matched cohort of patients with ADHD. For the patients with JIA, uveitis was the most prevalent co-existing autoimmune disease in both age groups. After uveitis, the most prevalent coexisting autoimmune disease among patients with JIA aged $<18$ years was chronic urticaria. In patients with JIA aged $\geq 18$ years, type 1 diabetes mellitus was the most prevalent. Conditions with similar rates among patients with JIA aged $<18$ and $\geq 18$ years included alopecia areata, chronic urticaria, Crohn's disease, Hashimoto's thyroiditis/autoimmune thyroid disease, and systemic sclerosis/scleroderma.

Among patients with JIA aged $<18$ years, across both databases the greatest ORs were seen for Sjögren's syndrome/sicca syndrome and uveitis (Table 2). Among these patients, similar odds were observed for pernicious anemia and primary biliary cirrhosis when compared with the matched patients with ADHD. Among patients with JIA aged $\geq 18$ years in MarketScan, the greatest ORs were recorded for uveitis; in PharMetrics, the greatest ORs were for uveitis and chronic glomerulonephritis.

Among patients of all ages with ADHD, the most prevalent autoimmune disease was chronic urticaria followed by type 1 diabetes. All autoimmune diseases studied were more prevalent among patients with ADHD aged $\geq 18$ years versus those $<18$ years, with the exception of celiac disease (Table 2). These data show an increase in the occurrence of autoimmune diseases in patients with ADHD aged $\geq 18$ years compared with those $<18$ years.

No greater odds in the ADHD cohorts versus the JIA cohorts were observed across pre-specified autoimmune diseases. 


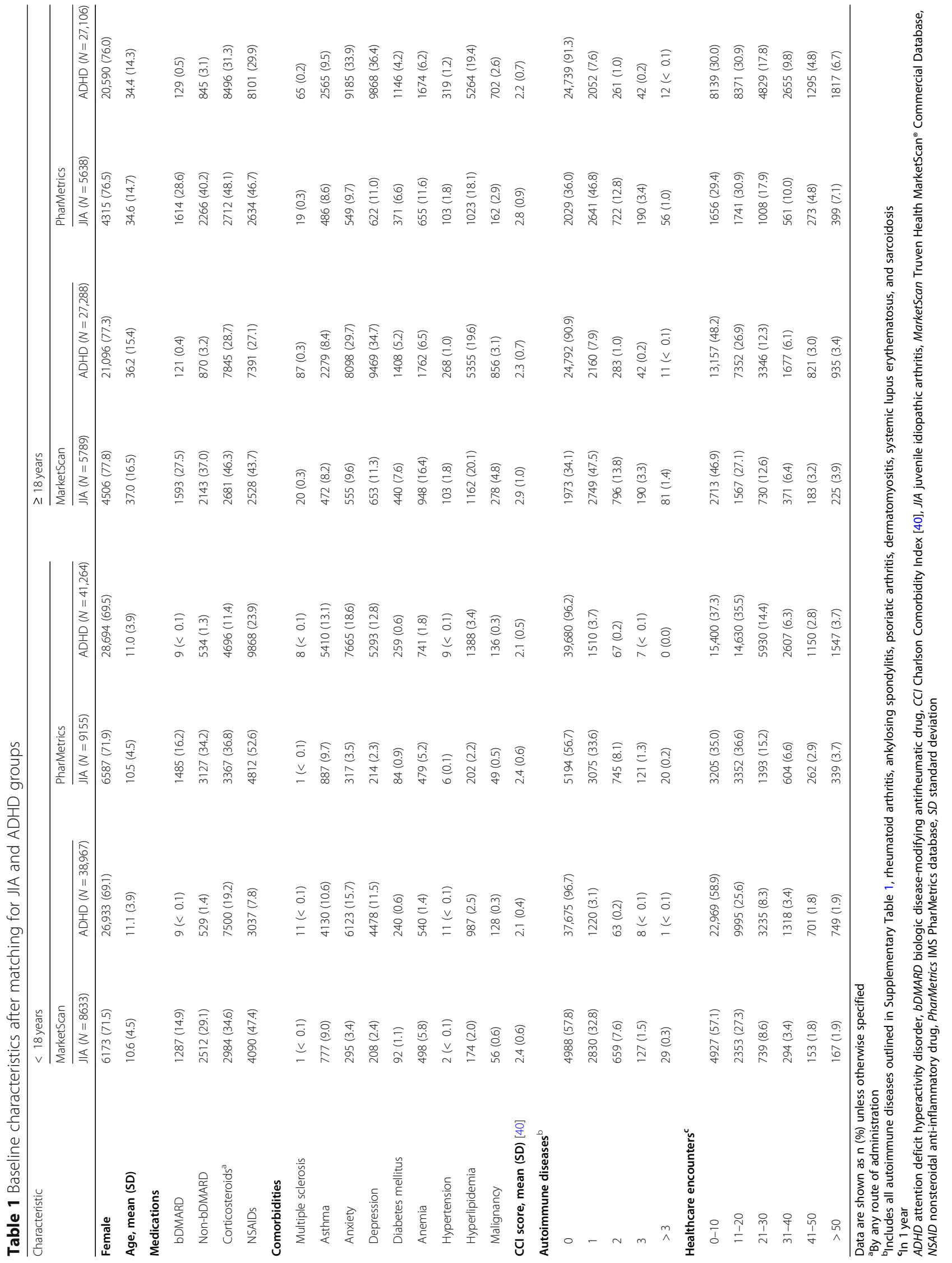




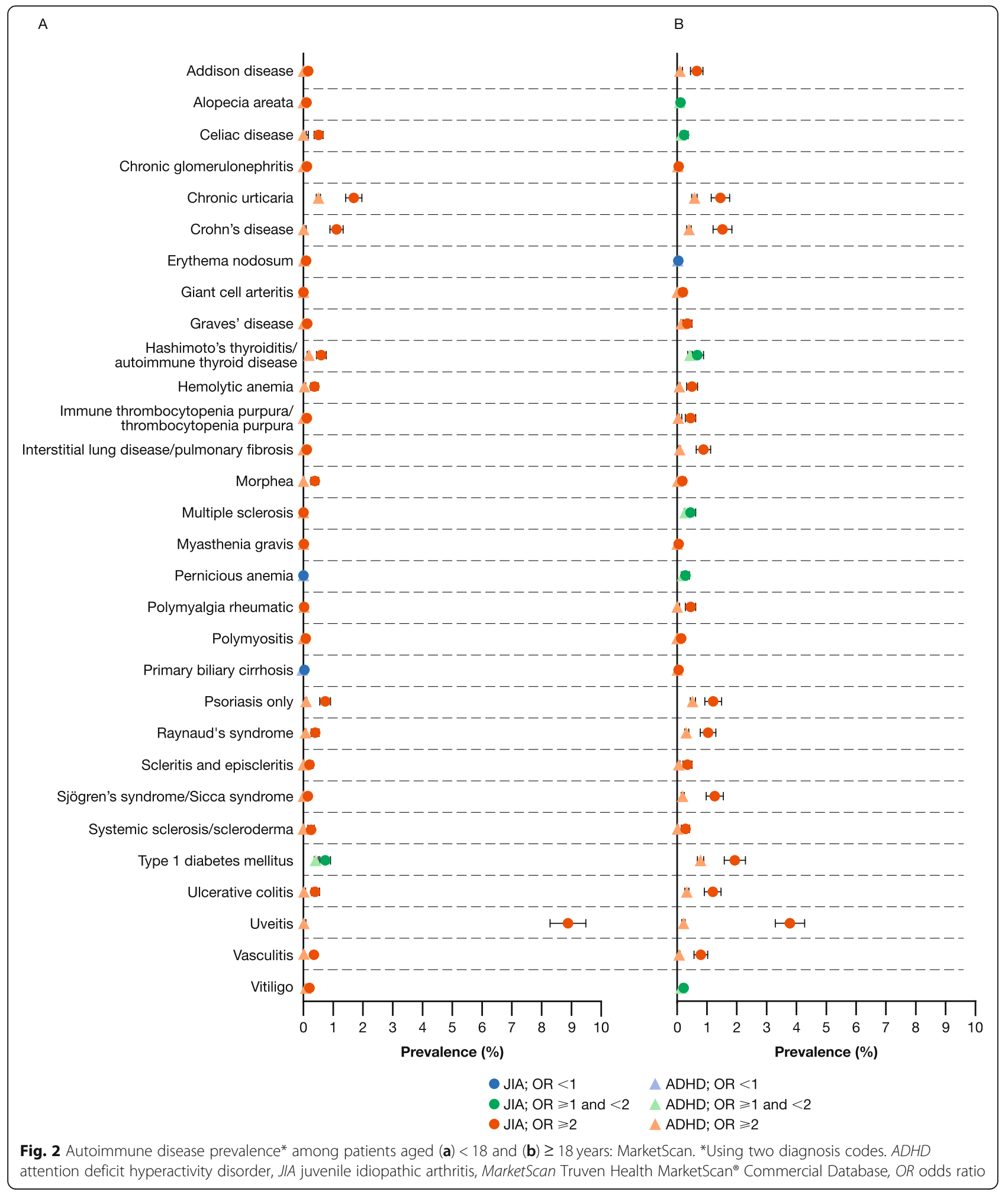

\section{Discussion}

This large, retrospective, cross-sectional study of data from two US healthcare claims databases evaluated 29, 215 patients with JIA and 134,625 matched control patients with ADHD aged $<18$ years and $\geq 18$ years. As expected, the findings suggest that autoimmune diseases are more prevalent in patients of all ages with JIA when compared to patients with ADHD. The results observed from both healthcare claims databases were largely similar. 


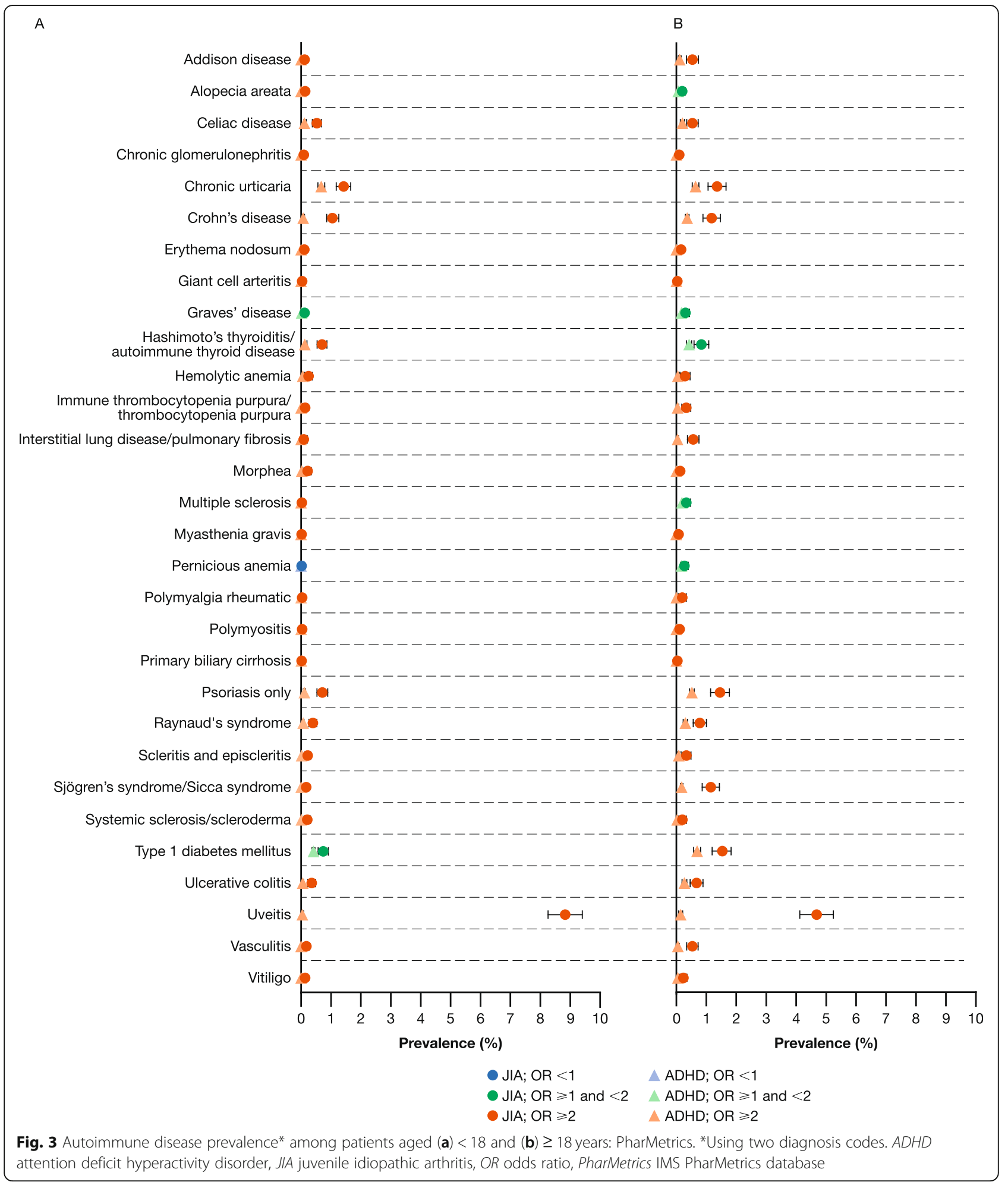

Patients with one autoimmune disease may be at a higher risk of developing a second one [43]. While a few studies have quantified the occurrence of co-existing autoimmune diseases in patients with JIA, to our knowledge, no large studies have been performed [2228]. In a single-center study in Italy of 79 patients with JIA (aged $\leq 21$ years), $15.2 \%$ of patients had at least one autoimmune disease in addition to JIA, with 


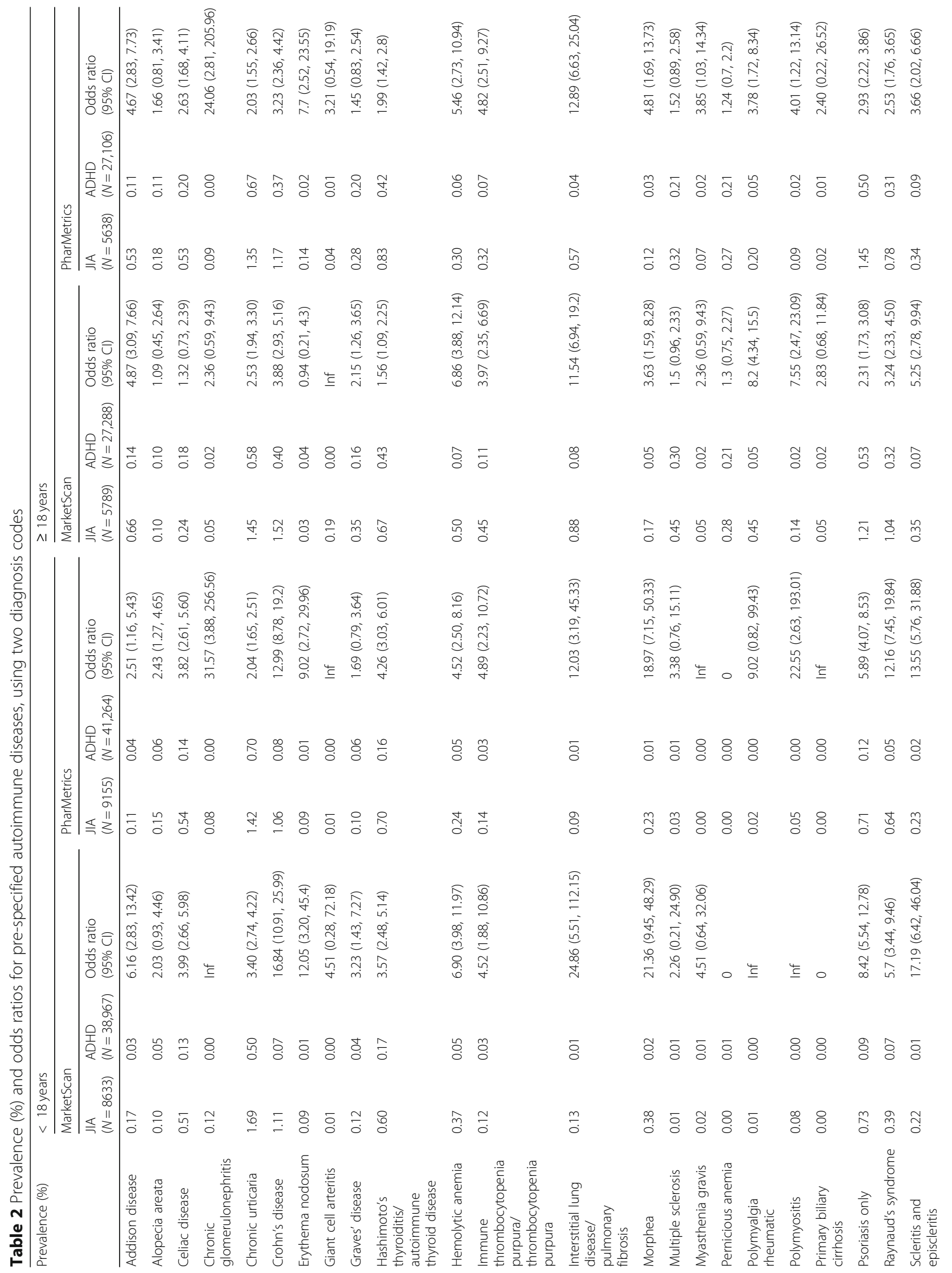




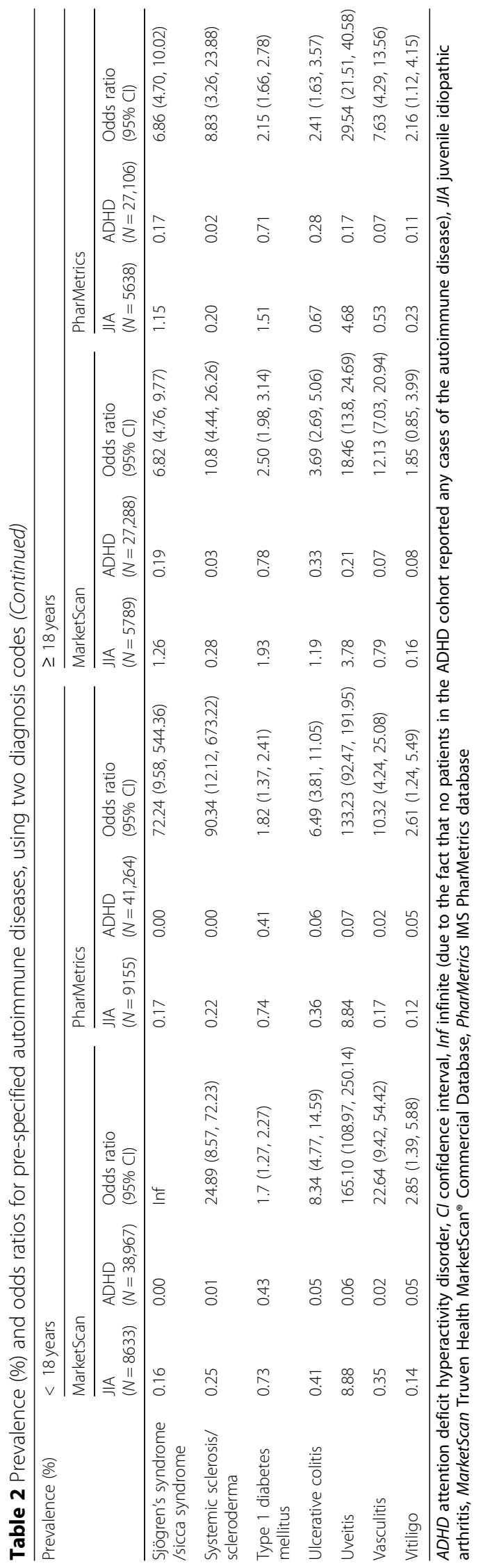


autoimmune thyroid diseases, such as Graves' disease, being the most common (10.1\%) [28]. Crohn's disease was present in $3.8 \%$ of patients with JIA in this study [28]. In another similar study in patients with JIA (aged $\leq 17$ years), $11.9 \%$ of patients had autoimmune thyroiditis and $6.6 \%$ of patients had celiac disease [22]. However, in this present study, autoimmune thyroiditis and celiac disease were reported in $<1 \%$ of patients, and Crohn's disease had a prevalence rate of $0.07-1.52 \%$. The differences observed between these studies and ours are likely due to variations in methodological approach: previous studies screened for co-existing autoimmune diseases by measuring thyroid functionality and autoantibodies in blood samples. In patients with positive autoantibody profiles, diagnosis of Crohn's disease or celiac disease was confirmed by a small bowel biopsy. Hence, these specific testing methods increase the likelihood of diagnosis of a co-existing autoimmune disease compared with reported ICD codes. However, consistently with the $\geq 18$-year-old cohort data reported in our study, a database study in Taiwan (patients with JIA, $n=$ 262; participants without JIA, $n=107,171$ ), quantifying the development of adult-onset rheumatic diseases among patients with JIA aged $\geq 16$ years, noted increased hazard ratios for ankylosing spondylitis, psoriasis, Sjögren's syndrome, SLE, and psoriatic arthritis [44].

In a prospective observational study investigating comorbidities in 344 adult patients enrolled in the Juvenile arthritis Methotrexate Biologics long-term Observation (JuMBO) registry, the following autoimmune conditions were reported to co-occur in patients with JIA: uveitis (17.7\%), psoriasis $(6.4 \%)$, Hashimoto's thyroiditis (1.2\%), Crohn's disease (1.2\%), ulcerative colitis $(0.9 \%)$, and diabetes mellitus $(0.9 \%)$ [32]. While the rates of gastrointestinal autoimmune diseases reported in JuMBO were comparable with those in our study, the rates of all other autoimmune diseases reported were higher in JuMBO. As noted above, the differences observed may be due to the way in which conditions were identified; patients from the JuMBO register were asked to self-report comorbid conditions and it has been noted that individuals may have different severity thresholds for reporting conditions. It is highly possible that comorbidity was overestimated by Raab et al. [32] as only severely affected patients were included, meaning the data were not representative of the general JIA population.

Although higher odds for the majority of autoimmune diseases were seen in the $<18$ years cohort compared with the $\geq 18$ years cohort (more patients with JIA than ADHD had a co-existing autoimmune disease), it must be noted that the prevalence rates in the older cohort were generally greater than those in the $<18$ years cohort (more patients with both JIA or ADHD had a coexisting autoimmune disease). This may reflect that the older patients had a greater amount of time to develop autoimmune diseases compared with the younger cohort prior to enrollment in this study. The large ORs observed in the patients aged $<18$ years can be attributed to the lower prevalence of autoimmune diseases among patients with ADHD compared with patients with JIA. These ORs indicate that patients with JIA are predisposed to the earlier development of these conditions.

Among patients with JIA, 23-54\% also had a diagnosis of RA. This is expected as, despite the fact the diseases are two distinct entities, non-pediatric rheumatologist physicians may give a diagnosis of RA, in particular when a patient with JIA moves from pediatric care to an adult healthcare setting $[41,42]$. A large, cross-sectional study investigating the prevalence of 37 autoimmune diseases in patients with RA or osteoarthritis revealed that patients with RA had a greater number of concurrent autoimmune diseases [15]. The most prevalent coexisting autoimmune conditions in the patients with RA were SLE and psoriatic arthritis. Diagnosis codes for both SLE and psoriatic arthritis were commonly reported in the JIA population of the present study, reflecting the similarities in the pathogenesis of JIA and RA. In the control osteoarthritis population, as in the ADHD population of our study, type 1 diabetes mellitus was the most highly prevalent co-existing autoimmune disease [15].

The co-existence of multiple autoimmune diseases in patients with JIA may be explained by the involvement of common genes. Autoimmune diseases are significantly more common among relatives of patients with JIA than among healthy controls [45], and a number of associations between human leukocyte antigen alleles and JIA, RA, celiac disease, and type 1 diabetes mellitus have been reported [46]. In addition, a study investigating the alleles and genotypes of patients with JIA and healthy controls revealed strong associations between JIA and variants in certain regions of TNFAIP3, STAT4 and C12ORF30 [47]. These regions have previously been associated with a number of other autoimmune diseases including RA and SLE [47].

The limitations of this study should be considered. Firstly, the ICD codes used in administrative data do not allow for distinction between the subcategories of JIA, which may have some influence on the prevalence of coexisting autoimmune diseases reported using these data. Although patients with JIA and ADHD may have a similar number of healthcare encounters, their primary providers are likely to be different (e.g. rheumatologist versus psychiatrist), which may introduce bias due to misclassification. Further misclassification bias may also have arisen due to diagnostic error caused by the complexity of polyautoimmunity, drug-induced symptom occurrence, or misdiagnosis. In addition, identification of 
medical events or baseline comorbidities here were restricted to computerized population-based data captured as part of the medical claims; these data can be challenging to verify. A study of hospitalized pediatric patients with rheumatic diseases in Kenya demonstrated 77\% agreement in coding between the treating physician and medical records clerk [48]. Similarly, a study in Germany demonstrated that two coders have ICD-10-code agreement in around $2 / 3$ cases [49]. It is also important to consider that autoimmune diseases are complex, diagnoses may change (e.g. arthritis associated with inflammatory bowel disease may initially be diagnosed as JIA if the arthritis presents before gastrointestinal symptoms) and symptoms often have multiple diagnosis codes. Finally, there is the possibility that some patients were included in both databases.

The large number of patients included in these analyses allowed for potential differences in the rates of coexisting autoimmune diseases between patients with JIA and patients with ADHD to be detected effectively. The use of two diagnosis codes reduces the risk that codes were entered in error or that a diagnosis was not confirmed. By including a range of possible diagnostic codes for each autoimmune disease, the likelihood of identifying the conditions from the claims data was maximized. The highly similar results observed between both healthcare claims databases also suggests a good degree of reliability.

\section{Conclusions}

We observed that patients with JIA had a greater prevalence of co-existing autoimmune diseases compared to patients with ADHD. In addition, the large ORs suggest that patients with JIA may be predisposed to the earlier development of autoimmune diseases than the general pediatric population. Management and treatment options for patients with JIA should be considered in the context of any other autoimmune diseases present. The design and analyses of future studies assessing the impact of treatment in patients with JIA need to incorporate the risk of other autoimmune diseases in this population.

\section{Supplementary information}

Supplementary information accompanies this paper at https://doi.org/10. 1186/s12969-020-00426-9.

\section{Additional file 1: Table S1. ICD-9-CM and ICD-10-CM codes for pre-} specified autoimmune diseases.

Additional file 2: Table S2. Baseline characteristics before matching for JIA and ADHD groups.

\section{Abbreviations}

ADHD: attention deficit hyperactivity disorder; DMARD: disease-modifying antirheumatic drug; ICD-9-CM: International Classification of Diseases, Ninth
Revision, Clinical Modification; ICD-10-CM: International Classification of Diseases, Tenth Revision, Clinical Modification; JIA: juvenile idiopathic arthritis; JuMBO: Juvenile arthritis Methotrexate Biologics long-term Observation; MarketScan: Truven Health MarketScan ${ }^{\oplus}$ Commercial Database; OR: odds ratio; PharMetrics: IMS PharMetrics database; RA: rheumatoid arthritis; SLE: systemic lupus erythematosus; US: United States

\section{Acknowledgments}

Professional medical writing and editorial assistance was provided by Lola Parfitt, MRes, at Caudex, and was funded by Bristol Myers Squibb.

\section{Authors' contributions}

TAS, DJL, HK, SS, and HIB contributed to study conception and design. TAS and GPH contributed to the acquisition of data. TAS, DJL, GPH, HK, SS, and HIB analyzed and interpreted data. All authors were involved in drafting the article or revising it critically for important intellectual content, and all authors approved the final version to be published. All authors had full access to all of the data in the study and take responsibility for the integrity of the data and the accuracy of the data analysis.

Funding

This study was funded by Bristol Myers Squibb.

\section{Availability of data and materials}

All data generated or analyzed during this study are included in this published article/as supplementary information files. Bristol Myers Squibb policy on data sharing may be found at https://www.bms.com/researchersand-partners/independent-research/data-sharing-request-process.html.

\section{Ethics approval and consent to participate}

The study was conducted in accordance with the International Society for Pharmacoepidemiology Guidelines for Good Pharmacoepidemiology Practices, applicable regulatory requirements and the principles of the Declaration of Helsinki. No new studies with human or animal subjects are reported in this article. Review and approval by ethics committees and patient informed consent were not required for this study.

\section{Consent for publication}

Not applicable.

\section{Competing interests}

TAS was an employee of Bristol Myers Squibb at the time of the analysis (former employee at present). GPH and SS are consultants for Bristol Myers Squibb. HK was an employee of Bristol Myers Squibb at the time of the analysis (former employee at present) and is a consultant for Bristol Myers Squibb. HIB has received consulting fees and/or speakers bureau fees from Boehringer Ingelheim, Bristol Myers Squibb, EMD Serono, Genentech, Janssen, Lilly, Novartis, Pfizer, Roche, R-Pharm, and UCB; and has received research funds from Pfizer. Cincinnati Children's Hospital Medical Center has received consulting fees and/or honoraria for the work of HIB from Abbott, Amgen, AstraZeneca, Boehringer Ingelheim, Bristol Myers Squibb, EMD Serono, GlaxoSmithKline, Hoffman-La Roche, Janssen, Lilly, Novartis, Pfizer, Takeda, UBC, and Wyeth Pharma. DJL's institution, Cincinnati Children's Hospital Medical Center, has received consulting fees and/or honoraria for the work of DJL from Amgen, Abbott, AstraZeneca, Boehringer Ingelheim, Bristol Myers Squibb, Celgene, GlaxoSmithKline, Hoffman-La Roche, Janssen, Novartis, Pfizer, Takeda, UBC, and Wyeth Pharma.

\section{Author details}

${ }^{1}$ Bristol Myers Squibb, Princeton, NJ, USA. ${ }^{2}$ Mu Sigma, Bangalore, India.

${ }^{3}$ Cincinnati Children's Hospital Medical Center, Cincinnati, OH, USA.

Received: 28 August 2019 Accepted: 3 April 2020

Published online: 05 June 2020

\section{References}

1. American Autoimmune Related Diseases Association Inc. Autoimmune Disease Statistics. Available from: https://www.aarda.org/news-information/ statistics/\#1488234386508-a9560084-9b69. Accessed 2019. 
2. Jacobsen DL, Gange SJ, Rose NR, Graham NM. Epidemiology and estimated population burden of selected autoimmune diseases in the United States. Clin Immunol Immunopathol. 1997;84(3):223-43.

3. Cooper GS, Bynum ML, Somers EC. Recent insights in the epidemiology of autoimmune diseases: improved prevalence estimates and understanding of clustering of diseases. J Autoimmun. 2009;33(3-4):197-207.

4. Lerner $A$, Jeremias $P$, Matthias $T$. The world incidence and prevalence of autoimmune diseases is increasing. Int I Celiac Dis. 2015;3(4):151-5.

5. Thomas SL, Griffith C, Smeeth L, Rooney C, Hall AJ. Burden of mortality associated with autoimmune diseases among females in the United Kingdom. Am J Public Health. 2010;100:2279-87.

6. French AR, Mason T, Nelson AM, O'Fallon WM, Gabriel S. Increased mortality in adults with a history of juvenile rheumatoid arthritis. Arth Rheum. 2001; 44(3):523-7.

7. The American Autoimmune Related Diseases Association. Autoimmune disease list. Available from: https:/www.aarda.org/diseaselist/. Accessed 2019.

8. Cho JH, Feldman M. Heterogeneity of autoimmune diseases: pathophysiologic insights from genetics and implications for new therapies. Nat Med. 2015;21(7):730-8.

9. Farh KK, Marson A, Zhu J, Kleinewietfeld M, Housley WJ, Beik S, et al. Genetic and epigenetic fine mapping of causal autoimmune disease variants. Nature. 2015;518(7539):337-43.

10. Kumarasamy MA, Sanfilippo FP. Breaking down silos: engaging students to help fix the US health care system. J Multidiscip Healthc. 2015;8:101-8.

11. Ravelli A, Martini A. Juvenile idiopathic arthritis. Lancet. 2007;369(9563):767-78.

12. Prince FHM, Otten MH, van Suijlekom-Smit LWA. Diagnosis and management of juvenile idiopathic arthritis. BMJ. 2010;341:c6434.

13. Bertilsson L, Andersson-Gare B, Fasth A, Petersson IF, Forsblad-D'elia H. Disease course, outcome, and predictors of outcome in a population-based juvenile chronic arthritis cohort followed for 17 years. J Rheumatol. 2013; 40(5):715-24.

14. Selvaag AM, Aulie HA, Lilleby V, Flato B. Disease progression into adulthood and predictors of long-term active disease in juvenile idiopathic arthritis. Ann Rheum Dis. 2016;75(1):190-5.

15. Simon TA, Kawabata H, Ray N, Baheti A, Suissa S, Esdaile JM. Prevalence of co-existing autoimmune disease in rheumatoid arthritis: a cross-sectional study. Adv Ther. 2017;34(11):2481-90.

16. Thierry S, Fautrel B, Lemelle I, Guillemin F. Prevalence and incidence of juvenile idiopathic arthritis: a systematic review. Joint Bone Spine. 2014;81 (2):112-7.

17. Angeles-Han ST, Pelajo CF, Vogler LB, Rouster-Stevens K, Kennedy C, Ponder $L$, et al. Risk markers of juvenile idiopathic arthritis-associated uveitis in the childhood arthritis and rheumatology research Alliance (CARRA) registry. J Rheumatol. 2013;40(12):2088-96.

18. Ravelli A, Felici E, Magni-Manzoni S, Pistorio A, Novarini C, Bozzola E, et al. Patients with antinuclear antibody-positive juvenile idiopathic arthritis constitute a homogeneous subgroup irrespective of the course of joint disease. Arthritis Rheumatism. 2005;52(3):826-32.

19. Agrawal S, Desai MP. Simultaneous occurrence of type I diabetes mellitus and juvenile rheumatoid arthritis. Indian Pediatr. 2003;40(6):568-71.

20. Marques VM, Carvalho SC, Antunes AM, Marques OA, Silva MH, Vieira MJ. Graves' disease associated with juvenile idiopathic arthritis. Rev Bras Reumatol. 2011;2011(5):2.

21. Nagy KH, Lukacs K, Sipos P, Hermann R, Madacsy L, Soltesz G. Type diabetes associated with Hashimoto's thyroiditis and juvenile rheumatoid arthritis: a case report with clinical and genetic investigations. Pediatr Diabetes. 2010;11(8):579-82.

22. Stagi S, Giani T, Simonini G, Falcini F. Thyroid function, autoimmune thyroiditis and coeliac disease in juvenile idiopathic arthritis. Rheumatology. 2005;44:517-20.

23. Robazzi TC, Adan LF, Pimentel K, Guimarães I, Magalhães Filho J, Toralles $\mathrm{MB}$, et al. Autoimmune endocrine disorders and coeliac disease in children and adolescents with juvenile idiopathic arthritis and rheumatic fever. Clin Exp Rheumatol. 2013;31:310-7.

24. Alpigiani MG, Cerboni M, Bertini I, d'Annunzio G, Haupt R, lester A, et al. Endocrine autoimmunity in young patients with juvenile chronic arthritis. Clin Exp Immunol. 2002;20:565-8.

25. Unsal E, Oren O, Salar K, Makay B, Abaci A, Ozhan B, et al. The frequency of autoimmune thyroid disorders in juvenile idiopathic arthritis. Turk J Pediatr. 2008;50(5):462-5.

26. Mihailova D, Grigorova R, Vassileva B, Mladenoa G, Ivanona N, Stephanov S, et al. Autoimmune thyroid disorders in juvenile chronic arthritis and systemic lupus erythematosus. Rheumaderm. 1999;455:55-60.
27. Pohjankoski H, Kautiainen H, Korppi M, Sovolainen A. Simultaneous juvenile idiopathic arthritis and diabetes mellitus type $1-$ a Finnish Nationwide study. J Rheumatol. 2012;39(2):377-81.

28. Tronconi E, Miniaci A, Pession A. The autoimmune burden in juvenile idiopathic arthritis. Ital J Pediatr. 2017:43:56.

29. Wu JJ, Nguyen TU, Poon KY, Herrinton $\sqcup$. The association of psoriasis with autoimmune diseases. J Am Acad Dermatol. 2012;67(5):924-30.

30. Marrie RA, Reider N, Cohen J, Stuve O, Sorensen PS, Cutter G, et al. A systematic review of the incidence and prevalence of autoimmune disease in multiple sclerosis. Mult Scler. 2015;21(3):282-93.

31. Gabriel SE, Michaud K. Epidemiological studies in incidence, prevalence, mortality, and comorbidity of the rheumatic diseases. Arthritis Res Ther. 2009;11(3):229.

32. Raab A, Sengler C, Niewerth M, Klotsche J, Horneff G, Zink A, et al. Comorbidity profiles among adult patients with juvenile idiopathic arthritis: results of a biologic register. Clin Exp Rheumatol. 2013;31(5):796-802.

33. Flendrie M, Vissers WH, Creemers MC, de Jong EM, van de Kerkhof PC, van Riel PL. Dermatological conditions during TNF-alpha-blocking therapy in patients with rheumatoid arthritis: a prospective study. Arthritis Res Ther. 2005;7(3):R666-76.

34. Dendrou CA, Bell II, Fugger L. A clinical conundrum: the detrimental effect of TNF antagonists in multiple sclerosis. Pharmacogenomics. 2013;14(12):1397-404.

35. Mannion ML, Xie F, Baddley J, Chen L, Curtis JR, Saag K, et al. Analysis of health care claims during the peri-transfer stage of transition from pediatric to adult care among juvenile idiopathic arthritis patients. Pediatr Rheumatol. 2016:14(1):49.

36. Matthews M, Nigg JT, Fair DA. Attention deficit hyperactivity disorder. Curr Top Behav Neurosci. 2014;16:235-66.

37. Beukelman T, Xie F, Chen L, Baddley JW, Delzell E, Grijalva CG. Rates of hospitalized bacterial infection associated with juvenile idiopathic arthritis and its treatment. Arth Rheum. 2012;64(8):2773-80.

38. Overhage JM, Ryan PB, Reich CG, Hartzema AG, Stang PE. Validation of a common data model for active safety surveillance research. J Am Med Inform Assoc. 2012;19(1):54-60.

39. International Society for Pharmacoepidemiology. Guidelines for Good Pharmacoepidemiology Practices (GPP). 2016. Available from: http:// pharmacoepi.org/resources/quidelines 08027.cfm. Accessed 7 July 2016.

40. Charlson M, Szatrowski TP, Peterson J, Gold J. Validation of a combined comorbidity index. J Clin Epidemiol. 1994;47(11):1245-51.

41. Wipfler K, Pedro, S, Shaw, Y, Schumacher, R, Simon, T, Dominique, A, Reinhardt, A, Michaud, K. Does diagnosis matter? Clinical distinctions between adult JIA and RA, and a characterization of patients with JIA reclassified as RA in adulthood. Atlanta: 2019 ACR/ARP Annual Meeting; 2019.

42. Gower T. All Grown up: juvenile idiopathic arthritis and rheumatoid arthritis: arthritis foundation. 2017. Available from: http://www.kidsgetarthritistoo. org/living-with-ja/daily-life/transition/juvenile-arthritis-transition-torheumatoid-arthritis.php. Accessed 2019.

43. Somers EC, Thomas SL, Smeeth L, Hall AJ. Are individuals with an autoimmune disease at higher risk of a second autoimmune disorder? Am J Epidemiol. 2009;169(6):749-55.

44. Yong SB, Huang JY, Chiou JY, Wei JC. Adult outcome of juvenile idiopathic arthritis: a nationwide population-based retrospective cohort study in Taiwan. Int J Rheum Dis. 2019;22(7):1283-8.

45. Prahalad S, Shear ES, Thompson SD, Giannini EH, Glass DN. Increased prevalence of familial autoimmunity in simplex and multiplex families with juvenile rheumatoid arthritis. Arthritis Rheum. 2002;46(7):1851-6.

46. Hersh AO, Prahalad S. Immunogenetics of juvenile idiopathic arthritis: a comprehensive review. J Autoimmun. 2015;64:113-24.

47. Prahalad S, Hansen S, Whiting A, Guthery SL, Clifford B, McNally B, et al. Variants in TNFAIP3, STAT4, and C12orf30 loci associated with multiple autoimmune diseases are also associated with juvenile idiopathic arthritis. Arthritis Rheum. 2009;60(7):2124-30,

48. Migowa A, Colmegna I, Hitchon C, Were E, Ng'ang'a E, Ngwiri T, et al. The spectrum of rheumatic in-patient diagnoses at a pediatric hospital in Kenya. Pediatr Rheumatol Online J. 2017;15(1):4.

49. Stausberg J, Lehmann N, Kaczmarek D, Stein M. Reliability of diagnoses coding with ICD-10. Int J Med Inform. 2008;77(1):50-7.

\section{Publisher's Note}

Springer Nature remains neutral with regard to jurisdictional claims in published maps and institutional affiliations. 